\title{
Recent Advancements in Nanomechanical Characterization Techniques and Associated Technologies
}

\author{
Oden L. Warren, S.A. Syed Asif, Arpit Dwivedi, Wei Che, and Thomas J. Wyrobek
}

Hysitron, Inc., Minneapolis, MN 55112

Nanoindentation [1] is a widely used technique for quantifying mechanical properties of microscale to nanoscale volumes of materials of relevance to shrinking technologies such as microelectronics, hard disk drives, and microelectromechanical systems. A particularly successful class of nanoindentation instrumentation is the scanning nanoindenter, which is capable of conducting scanning probe microscopy using the indentation probe as the imaging stylus. Although extracting elastic modulus and hardness from nanoindentation tests does not require direct observation of the residual indentation impression for the purpose of measuring its size, the ability to visualize surfaces in the sub-optical regime has proven to be empowering nonetheless. Intimate knowledge of the position of the indentation probe in relationship to specific surface features enables site-specific mechanical characterization of engineered microstructures, individual grains and phases of materials, and nanoparticles, as examples. Moreover, re-examination of the surface after completing the nanoindentation test can provide valuable insight into the deformation mechanism at hand.

In recent years, nanoindentation technology has evolved from relatively simple instruments dedicated solely to quasistatic operation (slow loading and unloading) to nanomechanical / nanotribological test platforms supporting nanoscratch and nanowear (or nanomachining) experimentation, plus a host of novel mixed-mode techniques. Techniques involving dynamic forces superimposed onto quasistatic forces include the nanoscale analogue of dynamic mechanical (thermal) analysis [2] for determining complex modulus (storage and loss components) as a function of depth, frequency, or temperature, and a method of complex modulus imaging [3] for differentiating material phases on the basis of their spatial distribution of complex modulus. Figure 1 shows loss tangent (ratio of loss modulus to storage modulus) in an image format for a phasesegregated polymer blend. For certain classes of materials (e.g., ceramics and metals), a more appropriate mixed-mode technique is in-situ acoustic emission monitoring of nanoindentation [4] for detecting high-speed deformation transients associated with the onset of plasticity, phase transformation, fracture, or delamination. The manner of controlling the indentation process also has evolved. Recent work in augmenting the familiar PID control algorithm with a predictive feedforward loop [5] has considerably improved the performance of both load-controlled and depthcontrolled operation.

For any important technique, the process of growing from infancy to maturity eventually leads to a major paradigm shift in usage. In the early years, nanomechanical testing typically involved a single sample mounted on the instrument and perhaps ten nanoindentation tests per sample. Today, nanoindenters are being exploited as screening tools in a number of combinatorial materials science workflows. Such high-throughput research environments demand reconsideration of the definition of a sample. For example, a ternary alloy library deposited onto a silicon wafer may possess over 500 unique compositions of the alloy system. Screening all compositions to an adequate level of statistical certainty, at a rate consistent with the concept of high-throughput experimentation, places extreme burden on the instrument to complete each full test cycle quickly without significantly 
degrading accuracy. A recent combinatorial investigation of low- $k$ dielectric films on silicon wafers [6] entailed 70000+ indentations placed on 12000+ unique spots of material over a period of eight months. This amounts to a full test cycle every five minutes assuming non-stop operation for the duration of the project.

Looking to the future, one might envision a proliferation of techniques only loosely based on nanoindentation capabilities. After all, nanoindenters can be thought of as accurate, repeatable, sensitive force-displacement devices at the simplest level, and these qualities surely can be exploited in other non-traditional ways. The complementary technique of atomic force microscopy still has advantages in terms of force sensitivity and mechanical bandwidth, but generally suffers in terms of accuracy and repeatability when used to probe material or structure mechanics. Improving force sensitivity into the picoNewton range (with an accompanying loss in load capacity) will be essential if the gap between nanoindentation and AFM is to be further narrowed. An ongoing effort in developing a force-displacement device for quantitative nanoindentation in a transmission electron microscope [7] is a substantial step towards probing materials at the fundamental level (with realtime observation of microstructure evolution) in a manner in keeping with stringent metrological principles.

\section{References}

[1] A.C. Fischer-Cripps, Nanoindentation, Springer-Verlag, New York, 2004.

[2] S.A.S. Asif et al., Rev. Sci. Instrum. 70 (1999) 2408.

[3] S.A.S. Asif et al., J. Appl. Phys. 90 (2001) 1192; Erratum 90 (2001) 5838.

[4] N.I. Tymiak et al., Acta Mater. 52 (2004) 553.

[5] O.L. Warren et al., Z. Metallkd. 95 (2004) 287.

[6] K. Chondroudis et al., "A combinatorial workflow for the rapid discovery and optimization of low dielectric constant materials", presented at 2003 MRS Spring Meeting, Session E8, Apr. 21-25, 2003, San Francisco, CA.

[7] U.S. Department of Energy SBIR Grant No. DE-FG02-04ER8397.

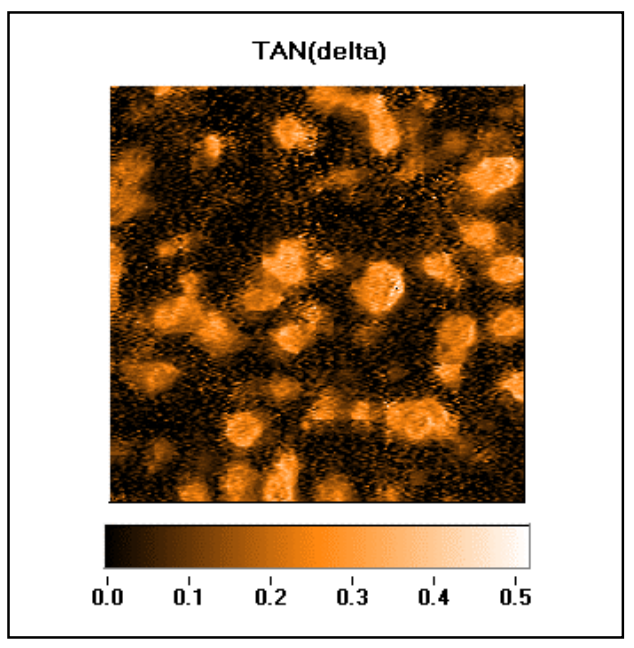

FIG. 1. Spatial distribution of loss tangent (ratio of loss modulus to storage modulus) for a phasesegregated polymer blend (10x10 $\mu \mathrm{m}^{2}$ image size). 